\title{
LOW-COHERENCE INTERFEROMETRY, AN ADVANCED TECHNIQUE FOR OPTICAL METROLOGY IN INDUSTRY
}

\author{
M. L. Dufour, G. Lamouche, V. Detalle, B. Gauthier, and P. Sammut
}

Industrial Materials Institute, National Research Council, Boucherville, Quebec, Canada

\begin{abstract}
Low-coherence interferometry (LCI) is an optical technique that may be used for industrial surface metrology with accuracy in the micron range. An instrument made with optical fibers is rugged enough to be used in industrial environments and the fiber- linked optical probe may be miniaturized for accessing tight locations. Among industrial applications developed at IMI we will present several cases for which LCI has been particularly useful such as an elongational rheometer (RME), characterization of wear damages on coating and Laser-induced Breakdown Spectroscopy (LIBS). The RME is an instrument in which a polymer sample is stretched in a controlled temperature furnace at up to $350^{\circ} \mathrm{C}$. LCI has been used for monitoring the thickness of the samples. Wear damage is quantified by the volume loss after a wear test. It requires a high depth resolution (axis perpendicular to the surface) but a relatively coarse transverse resolution. The LIBS is a technique that has been used for analyzing the chemical composition of materials as a function of depth. LCI has been integrated to a LIBS instrument for measuring accurately the crater depth between each laser shot
\end{abstract}

Introduction: Low-Coherence Interferometry (LCI) is an optical technique that relies on "coherence gating" to provide precise axial positioning of an object in the direction of light propagation. By focusing the light in a sample, one also obtains a good transverse resolution (perpendicular to the optical beam), thus collecting information from a finite volume for imaging or optical characterization purposes. This application has evolved at a fast pace in the last decade especially for the characterization of biological tissues under the field of Optical Coherence Tomography (OCT). Significant achievements were reported both for the underlying technology (optical sources, scanning systems) and the diagnostic capabilities. Successful applications of OCT have been reported in various medical fields like ophthalmology, dermatology and cardiology. ${ }^{1}$ LCI is also full of promises for industrial applications, a field that is not yet fully exploited. One of the few examples found in the literature is the measurement of the level of molten glass with a temperature over the surface in the range of $800^{\circ} \mathrm{C}^{2}$

A LCI system can provide precision of the order of microns, both in the axial and transverse positions. A fiber-based system where the light is brought to the sample through a single mode fiber allows isolating the measurement location from the system main components at a distance of meters or even hundreds of meters. There is no direct contact between the sample and the fiber output, thus allowing measurements on samples at high temperature. A system can thus be easily adapted to industrial conditions even in a harsh environment and tight location. A small optical head can be brought close to the sample for illumination purpose while the sensitive electronics is kept away in a more suitable environment.

In this paper, we review industrial applications of LCI recently developed at the Industrial Materials Institute (IMI). Firstly, LCI is used to monitor the thickness and width of polymer sample being stretched in an elongational rheometer. Secondly, LCI provides information about the volume loss in a wear test. Thirdly, LCI is combined with Laser Induced Breakdown Spectroscopy (LIBS) to provide chemical information as a function of depth in a material. These applications pertain to different industrial fields and should give an appreciation of the large potential of LCI to address industrial challenges.

Principles of LCI: The optical configuration of a typical LCI system based on fiber optics is illustrated in Fig.1. A single 2x2 optical fiber coupler is used to implement a Michelson interferometer. The power of a low-coherence source, typically a superluminescent diode, is divided into the sample arm and the reference arm of the interferometer through the fiber coupler. The light reflected by the reflectors in the sample is recollected by the sample arm fiber. The light reflected by the reference mirror is recollected by the reference arm fiber. The light from both the sample and reference arms is again coupled back in the fiber coupler and part of it is redirected towards the detector. Due to the finite coherence length of the source, optical interference is observed only when the optical pathlengths of the beams reflected by the sample reflector and the reference mirror differ from less than the coherence length.

During a measurement, the reference mirror is scanned. Figure 2 presents a typical signal recorded on the detector for a single reflector in the sample arm as a function of the optical path difference. The signal is composed 
of a continuous background on which is superimposed an oscillatory pattern resulting from successive constructive and destructive interferences. The interference pattern is modulated by an envelope function related to the coherence function of the source. It is a common practice to consider only the envelope function and, using a wellcalibrated system, the position of a reflector in the sample arm is determined by the center of the envelope function. For a source with a gaussian spectrum centered on wavelength $\lambda_{0}$ with a spectral width $\Delta \lambda$, the resolution provided by the envelope function is $\Delta z=2 \ln (2) \lambda^{2} /(\pi \Delta \lambda) .{ }^{1}$ A superluminescent diode provides a typical resolution of a few tens of microns. In the case of a sample containing well-spaced reflectors, a defined envelope function is recorded for each reflector. The position of the reflector can be determined more precisely by locating the exact position of the maximum of the envelope function, providing submicron resolution. This is often encountered in industrial applications.

The above discussion focuses on the axial resolution provided through what is called "coherence gating" in low coherence interferometry. The transverse resolution (perpendicular to the optical axis) is provided by the focusing optics in the sample arm. It is a common practice to rely on high quality optical components to work with diffraction limited spot sizes. The exact choice of components is application specific, being a trade-off between spot size and depth of focus.

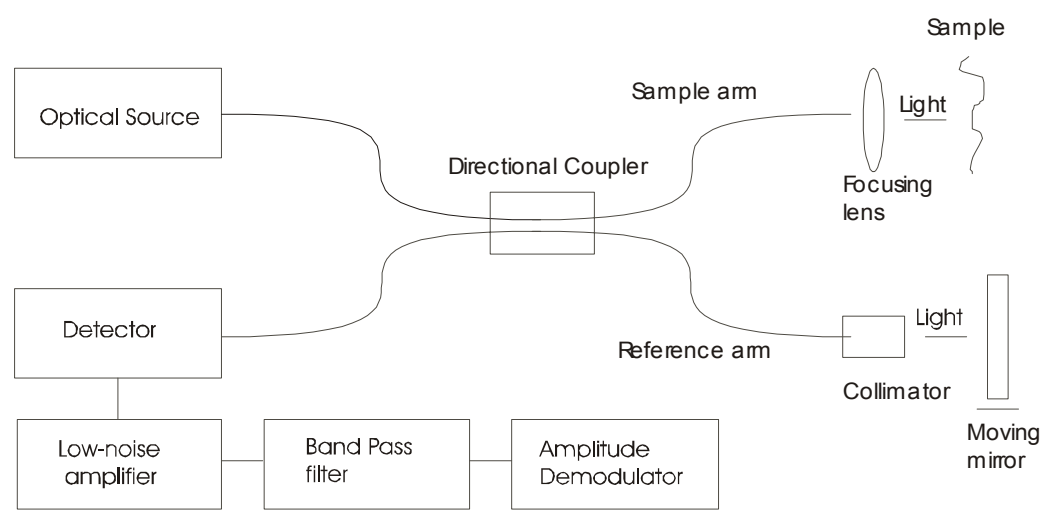

Figure 1: Typical configuration of a fiber-based system for low coherence interferometry

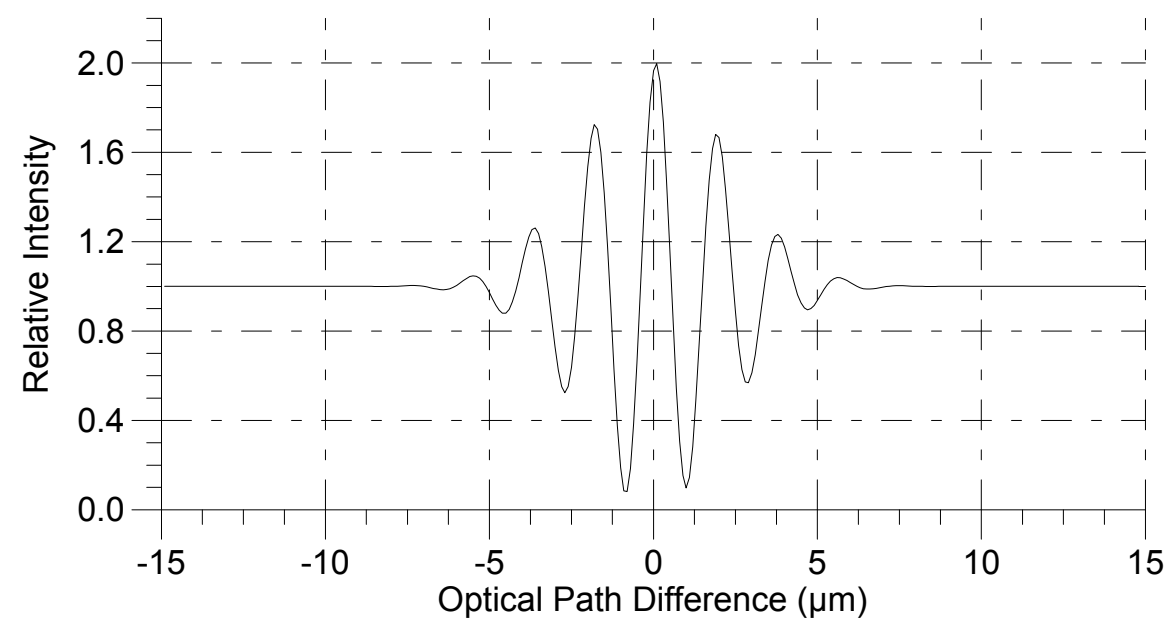

Figure 2: Interference signal recorded from a single reflector as a function of the optical pathlength difference. 
Elongational Rheometry: The relevance of elongational flow properties of polymers to industrial processes such as film blowing and foam extrusion is now well being recognized. The rotary clamp elongational rheometer, built around a counter-rotating belts principle use to stretch a polymeric material in the molten state, enables precise determination of the flow properties under controlled experimental conditions. The underlying experimental concept is based on the constant gauge length method where a specimen is subjected to an uniaxial deformation under a constant strain rate. ${ }^{3}$ In short, this constant gauge length rheometer draws the specimen at fixed points in space, maintaining the specimen length constant but changing its volume. The challenge is to accurately monitor, in real time, the volume change. This kind of measurement is very delicate, especially since very high strains can be achieved (more than 1000 times the initial length), and the material deformation must be carefully monitored to evaluate the true strain rate. The strain rate can be inferred by imaging the sample shape or a pattern at the surface of the sample. This is nevertheless a tedious and non-efficient approach. ${ }^{4}$

LCI is very well suited for this task since polymeric materials are translucent to visible and near-infrared light. LCI is non-contact and can easily be applied to a sample heated at a few hundred degrees Celsius as in the case of a Polymer Melt Elongational Rheometer for Melts (RME). The experimental setup is illustrated in Fig. 3, in which the sample is stretched using rotary belts in the RME apparatus. The output of the sample arm fiber is focused in the sample region. A rotating mirror is used to scan the sample along its width. The front and rear faces of the polymer film provide two peaks in the LCI measurement from which the optical thickness $T_{\text {opt }}$ between the two polymer faces is evaluated. It is related to the true geometrical thickness $T$ and the film refractive index $n$ by $T_{\text {opt }}=n T$. If the refractive index is unknown, the true thickness can still be obtained by measuring the position of a backward reference surface with and without the film, the difference in optical distance being given by $\Delta D=(n-1) T$. It is then quite straightforward to obtain the true thickness of the film $T=T_{o p t}-\Delta D$ and its refractive index $n=T_{o p t} / T$. The width of the film is obtained by displacing the spot from one side of the sample to the other with the rotating mirror, locating the extrema positions at which the reflections from the polymer film disappear.

LCI can thus provide accurate real-time measurements of the geometrical deformation of the film. This information can then be used to evaluate precisely the true strain rate applied to the film and improve the accuracy of the measurements performed with the elongational rheometer. Figure 4 presents the comparison between the true strain rates in an RME measurement evaluated with the LCI approach (lines) and with a more conventional video imaging technique. A LCI system is now permanently installed and used on the RME device at IMI. 


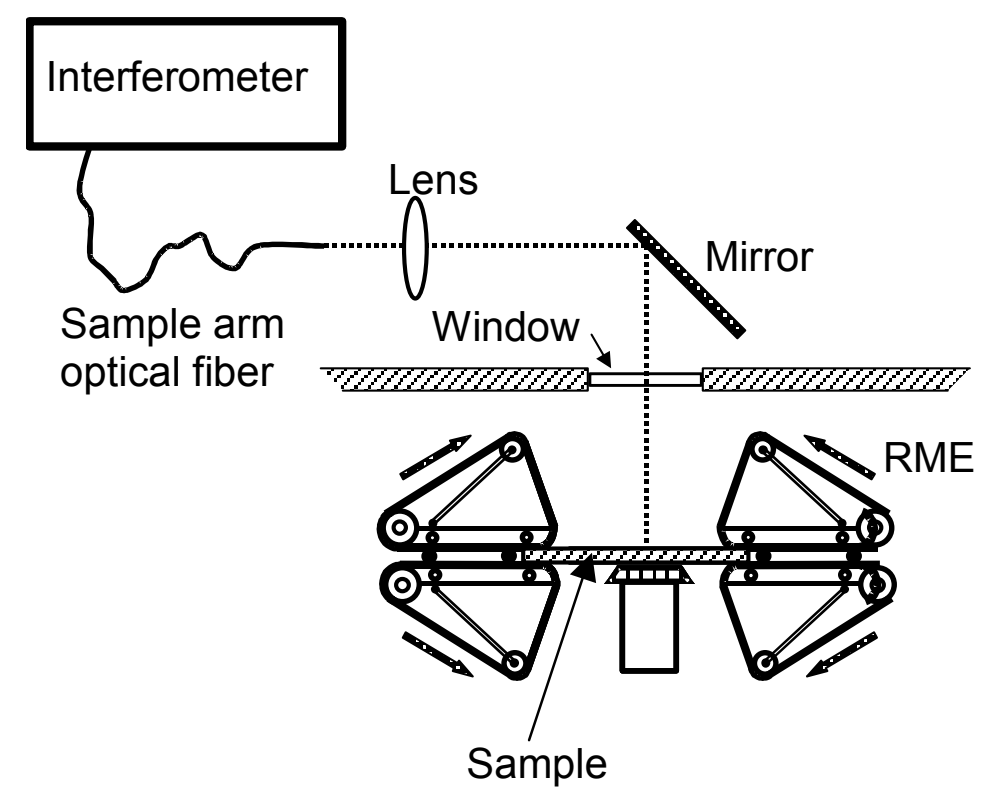

Figure 3: Experimental setup for LCI characterization of the sample deformation in a Rheometric Elongational Rheometer for Melts (RME).

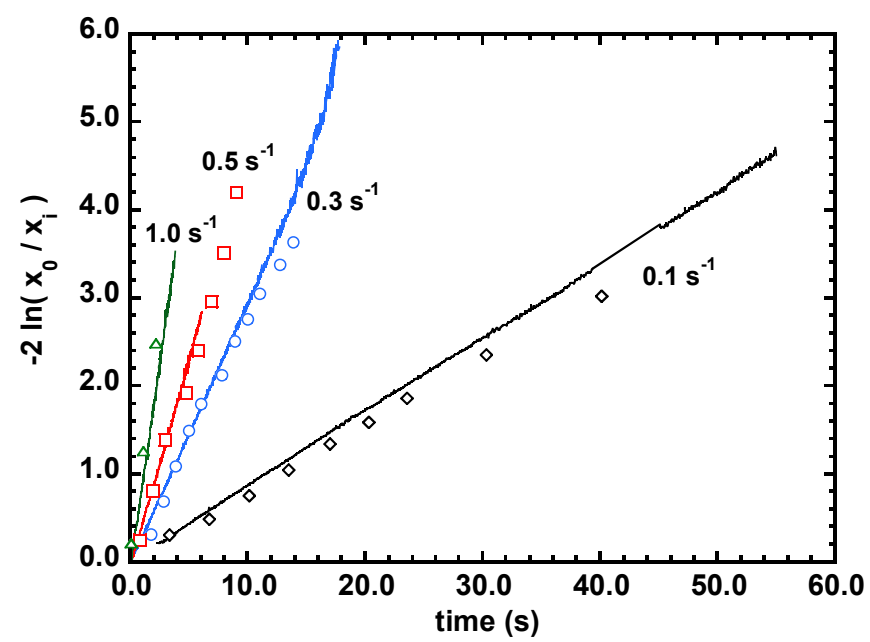

Figure 4: Comparison between the true strain rates evaluated using LCI (lines) and using a more conventional technique using video imaging (open symbols). The strain rates are evaluated from the variation $\left(\mathrm{x}_{0} / \mathrm{x}_{\mathrm{I}}\right)$ of the thickness (LCI) or width (video imaging) of the sample with time.

Wear test: The accuracy provided by LCI measurements makes it an ideal candidate for profilometry. When the sample presents a well-defined interface, a resolution of the order of a micron can be obtained even with a superluminescent source with a coherence length in the tens of microns since the center of the envelope function can be precisely determined. One such application developed at IMI is volume loss determination in a wear test. A surface map is obtained by scanning LCI measurements over the damaged region. The scanned surface must 
exceed the damaged region since an intact surface around it is necessary for reference purpose. The volume loss is evaluated by first determining the reference profile corresponding to an intact region. Then, each point measured on the damaged surface is compared to this reference surface and the measured differences are summed. Typically, a surface of $10 \mathrm{~mm}$ X $10 \mathrm{~mm}$ must be scanned for erosion tests, while $20 \mathrm{~mm} \mathrm{X} 50 \mathrm{~mm}$ are required for abrasion test with a rubber wheel. The required transverse resolution is not too severe, being about 100 microns $\mathrm{X} 100$ microns. But the resolution in depth (axial resolution) must be of the order of a micron since the maximum depth measured in a wear test is of the order of 10 microns. Figure 5 presents the results of a LCI characterization of an erosion test. A maximal depth of 8 microns was measured and the total volume loss was evaluated to $0.03 \mathrm{~mm}^{2}$.

IMI developed a robust and low-cost prototype for this application. Our first client was our Surface Technologies group who has successfully used the system for more than 4 years. A system was also delivered to Syncrude Canada Ltd.
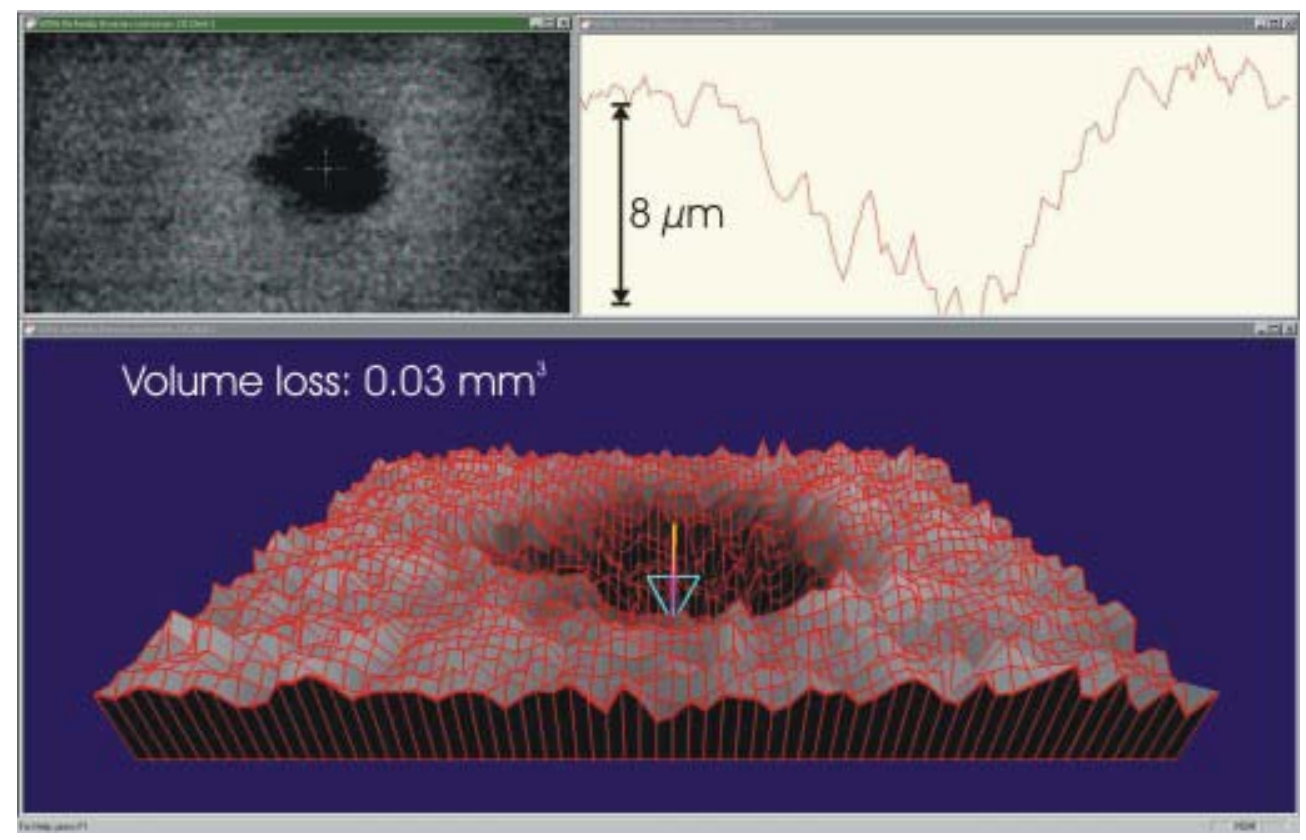

Figure 5: Profilometry of a surface after a wear test as measured by LCI.

Laser-Induced Breakdown Spectroscopy: The Laser-Induced Breakdown Spectroscopy (LIBS) is a powerful technique for rapid on-site analysis of solid, liquid, or gas. A powerful laser beam is focused on a sample, some matter is vaporized and a hot spark (plasma) is formed. Light emitted by the plasma is composed of spectral lines characteristic of the elements present in the sample. By spectrally analyzing this light, it is possible to deduce the elemental composition of the material under study. Moreover, the proportionality of spectral line intensity to elemental concentration enables quantitative analysis. The detection limit of the technique is in the ppb range.

In a solid, the concentration of an element can be traced as a function of the depth in the material by continuously firing the laser at the same spot. The detph can be estimated by first calibrating the amount of volume vaporized for each pulse. But this is a tedious process that asks for different calibration curves for different materials and different operating conditions.

LCI is very well suited to complement the LIBS apparatus to provide depth information. A typical LIBS crater is $1 \mathrm{~mm}$ in diameter and the LCI beam can easily reach the bottom of the crater to provide depth information. Figure 6 illustrates the combination of a LCI system with a LIBS system. Only the illumination part of the sample is shown. The LIBS system operates with a laser beam at 1.06 microns focused on the sample surface. The LCI system operates around 1.3 microns, thus, the output of the sample arm fiber can be combined with the LIBS beam with a dichroic mirror. Prior to the dichroic mirror, the LCI beam is deflected by a rotating mirror that allows scanning of the LIBS crater along a line. It is more efficient to observe also the surroundings of the crater to obtain 
a more accurate estimate of the crater depth. Typically, for a crater of $1 \mathrm{~mm}$ diameter, the LCI measurement is performed along a $3 \mathrm{~mm}$ line.

Figure 7 shows the measured depth as a function of the number of laser pulses in a steel sheet with a zinc coating. It takes about 140 pulses to dig through the zinc layer for which the removal rate is about $55 \mathrm{~nm} / \mathrm{pulse}$. In steel, the removal rate is much lower and is about $7 \mathrm{~nm} /$ pulse. Figure 8 shows the concentration of zinc as a function of depth for a steel sample coated with Zinc. The results of two LIBS configurations are shown (different ablation spots), and in both case, LCI is used to measure the depth. This can be used to measure the thickness of the Zinc layer. This demonstrates the ability of the LCI system to complement the LIBS apparatus in providing depth information.

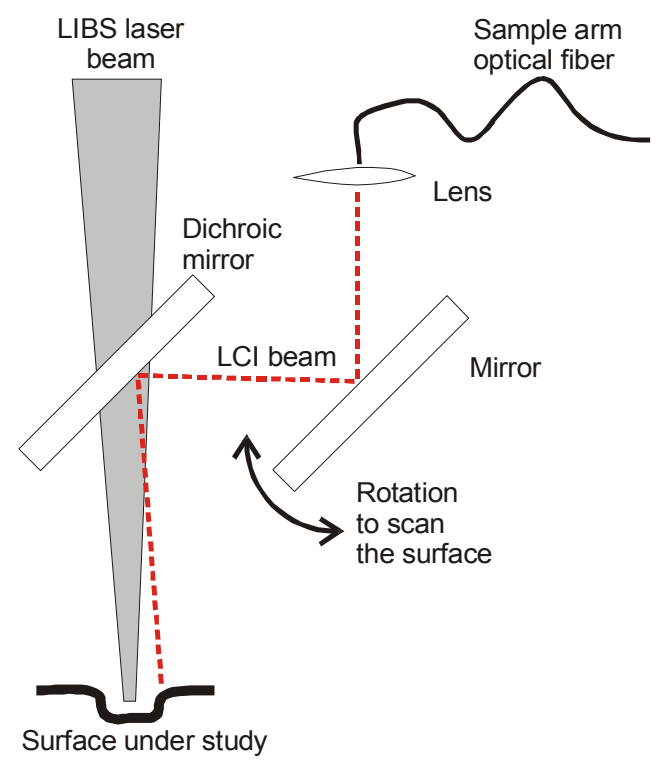

Figure 6: Illumination of the sample for simultaneous LIBS and LCI measurements.

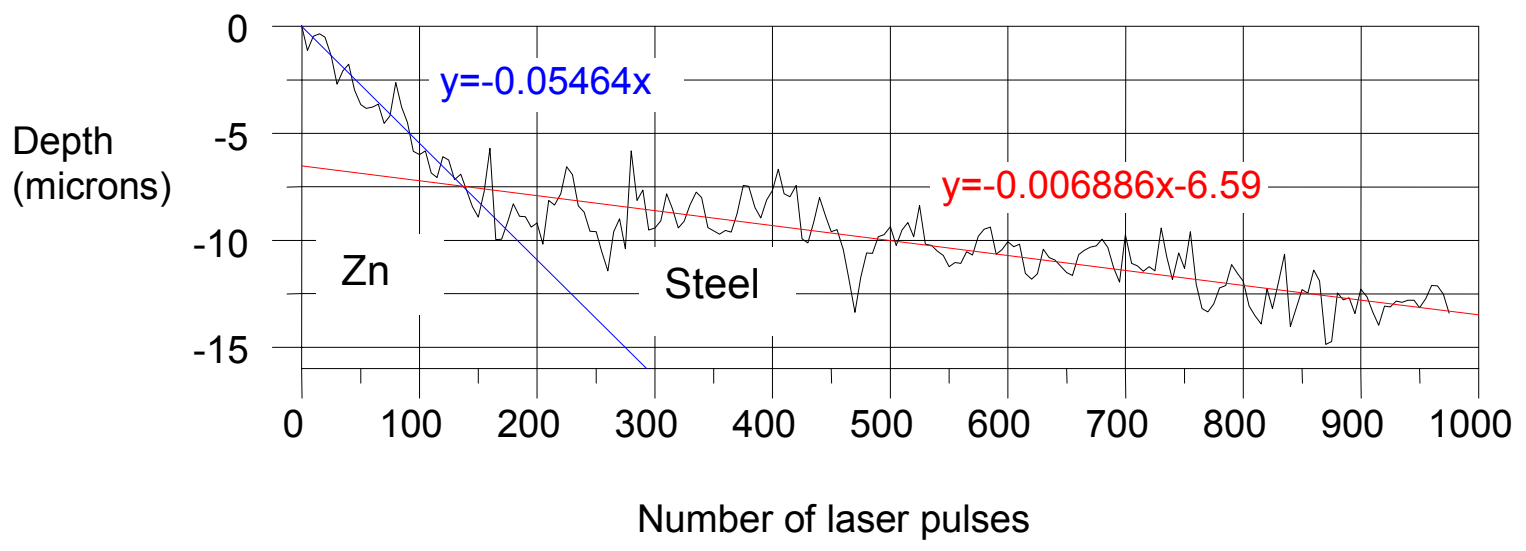
with zinc.

Figure 7: Depth as a function of the number of laser pulses as measured by LCI for steel sample coated 


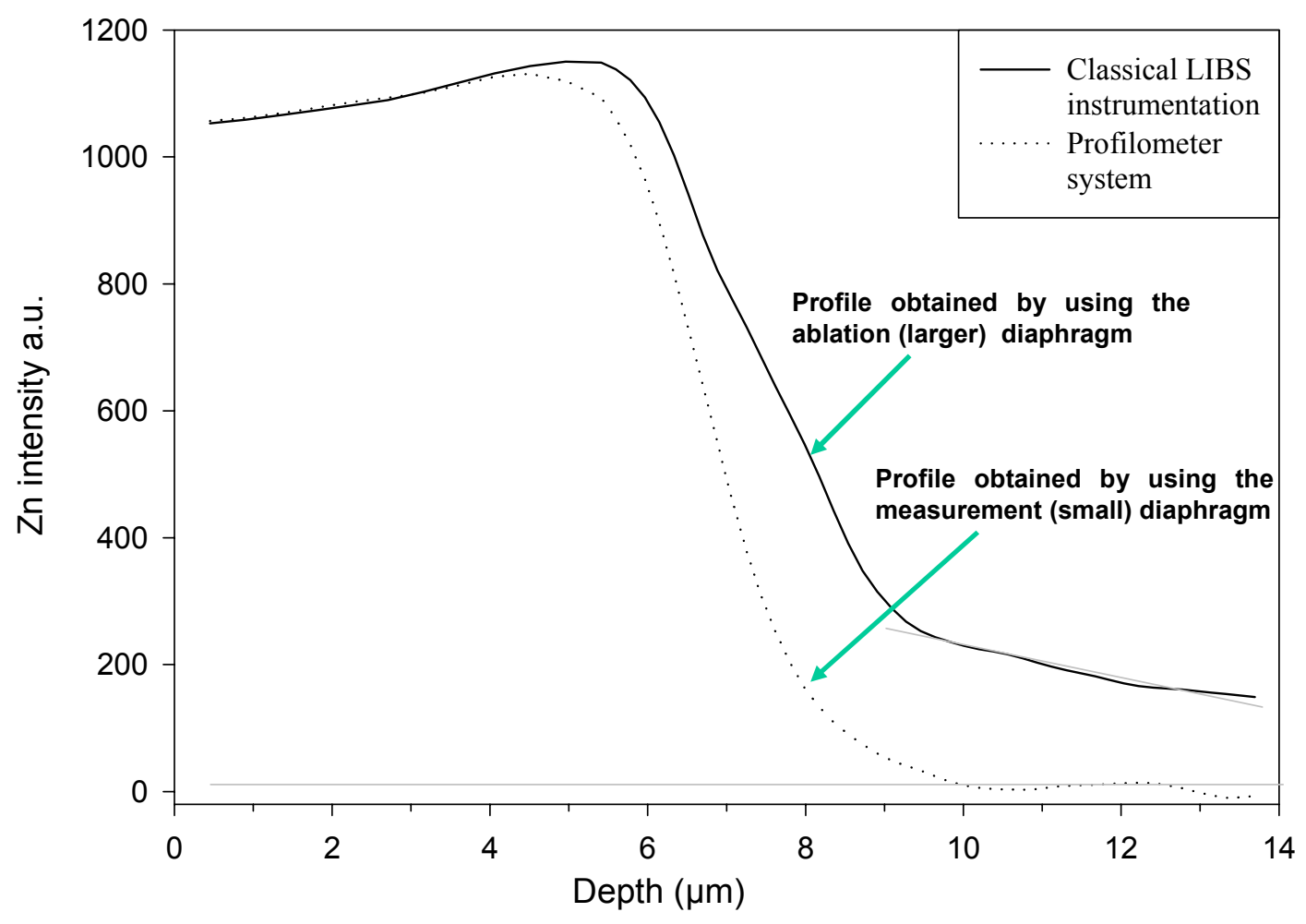

Figure 8: LIBS concentration of Zinc as a function of depth for a steel sample coated with Zinc. Different spot sizes for LIBS measurements are compared. In both case, the depth is measured with LCI.

Conclusion: We have reviewed a few applications of LCI for industrial applications developed at IMI over the recent years. LCI is a non-destructive and non-contact technique. It can thus be applied on high temperature samples. It is highly adaptable to various industrial conditions since only the optics of the sample arm output needs to be close to the sample. The other components of the interferometer can be put at distances as high as hundreds of meters if one uses a fiber-based delivery. In vivo imaging of the interior walls of an artery have been demonstrated in biomedical applications, industrial applications of LCI in tight locations should thus be easily achieved. The LCI technique is versatile enough to be coupled with another technique to improve the precision of the latter or enlarge its functionality. It is a technique that is full of potential for a variety of industrial applications and we should see more of these applications over the forthcoming years.

\section{References:}

1. B. E. Bouma and G. J. Tearney, eds., Handbook of Optical Coherence Tomography (Marcel Dekker, New York, 2002).

2. L. Giniunas, R. Karkockas, and R. Danielius, "Accurate remote distance sensing by use of lowcoherence interferometry: an industrial application," Applied Optics 37(28), 6729-6733 (1998).

3. T. Schweizer, "The uniaxial elongational rheometer RME - six years of experience," Rheologica Acta 39(5), 428-443 (2000).

4. R. Gendron, P. Sammut, M. Dufour, and B. Gauthier, "Low-coherence interferometry applied to uniaxial elongational rheometry," presented at the Annual Technical Conference ANTEC, Conference Proceedings, 2003. 